\begin{tabular}{|l|l|c|l|}
\hline Eiszeitalter u. Gegenwart & 43 & $\begin{array}{c}29-43 \\
\text { 6 Abb., 1 Beil. }\end{array}$ & Hannover 1993 \\
\hline
\end{tabular}

\title{
Zur erdgeschichtlichen Entwicklung der Emsland-Moore
}

\author{
Simone Hauschild \& GeRd LÜTTIG*)
}

NW Germany - Emsland - peat-bog - Holocene history of vegetation

\begin{abstract}
Kurzfassung: Im Zuge der geologisch-telmatologischen Untersuchung des geplanten Prüfgeländes der MercedesBenz AG in Papenburg sind zahlreiche Bohrungen in sehr dichtem Abstand in den Mooren südöstlich von Papenburg durchgeführt worden. Die dortigen Hochmoore (Wildes Moor, Kortemoor und Klostermoor) erwiesen sich als z. T. wurzelecht, z. T. vom Versumpfungsmoortyp mit Schwarztorf-Auflage auf Mudden, lokal auch auf Niedermoor- und Bruchwaldtorf. Eine enge Bindung der Vermoorung an Ortsteinhorizonte einer frühpostglazialen Podsolierung, welche sowohl über den Talsanden als auch den Älteren Flugsanden ausgebildet ist, ist festzustellen. Die Hochmoortorfbildung setzt üblicherweise erst in der zweiten Hälfte der Pollenzone VII, gleichzeitig mit der Neolithischen Revolution ein. Starke Beteilung von Eriophorum ist für die Schwarztorfe typisch. Die der Leda und Ems tributären Nebenflüsse zeigen eine spätglazial-frühpostglaziale, auf den spätweichselzeitlichen tiefen Meeresspiegel bezogene Einschneidungsphase in die weichselzeitliche Talsandebene. Mit dem postglazialen Meeresspiegelanstieg schritt die Akkumulation - auf die ansteigende Vorflut eingespielt - von den Flußunterläufen aufwärts fort. Junge Dünenzüge begleiten die für die Siedlungsgeschichte wichtigen Talsandterrassenränder, hinter denen das Sphagnentorfwachstum bis in die Weißtorffazies fortschritt.
\end{abstract}

\section{[Stratigraphical development of the Emsland peat-bogs (NW Germany)]}

\begin{abstract}
In the peat-bogs southeast of Papenburg numerous drillings, all only a short distance apart, were carried out as a part of the geological-telmatological study of the testing range planned by Mercedes-Benz in Papenburg. The bogs located there (Wildes Moor, Kortemoor and Klostermoor) can be grouped partly into autochthonous bogs, partly into paludigeneous bogs with a black layer of peat on top of mud deposits, at specific sites starting with low moor peat or forest-swamp peat. Peat formation is closely linked to the occurence of iron pans of an early post-glaci-
\end{abstract}

\footnotetext{
*) Anschrift der Verfasser: Frau Simone Hauschild, Institut für Palynologie und Quartärwissenschaften der Universität Göttingen, Wilhelm-Weber-Str. 2, 3400 Göttingen. Prof. Dr. GERD LÜTTIG, Institut für Geologie und Mineralogie der Universität Erlangen, em. o. Prof. für Angewandte Geologie, Schloßplatz 5, 91054 Erlangen

Nach einem von G. LÜTTIG am 22.05.1991 vor der Arbeitsgemeinschaft NW-deutscher Geologen in Bad Bentheim gehaltenen Vortrag.
}

al podzolization. This is particularly evident in the Lower Terrace river sands (Talsande) as well as in the older eolian sands. High-moos peat formation normally set in the second half of pollen zone VII and coincided with the Neolithic Revolution. Typical of black high-moos peat is the high percentage of Eriophorum. Tributaries of the rivers Leda and Ems show a Late Glacial-Early Postglacial incision into the Weichselian valley sands which is dating back to the late Weichselian deep sea-level. With the Postglacial rise of the sea level accumulation - correlating with the rising tides - spread river-upwards from the lower course of the rivers. Young chains of dunes stretch along the Lower Terrace margins, which were so important to colonization, whereas on the inland side Sphagnum vegetation thrived up to the white high-moos peat facies.

\section{Technische Vorbemerkungen}

Der zweitgenannte Autor führte für die Firma Mercedes-Benz AG, Stuttgart, eine geologisch-moorkundliche Kartierung der Moore südöstlich von $\mathrm{Pa}$ penburg/Emsland (Wildes Moor, Kortemoor und Klostermoor, Abb. 1) im Rahmen der Voruntersuchung der Brauchbarkeit des betreffenden Gebietes als Prüfgelände durch. Studenten des Geologischen Institutes der Universität Erlangen-Nürnberg teuften dazu ca. 1700 Handbohrungen bis zur Torfbasis ab. In Zusammenarbeit mit den Ingenieurbüros IGI, Dr. hc. Niedermayer, Westheim, und Grundbaulabor Bremen, Dipl.-Ing. BEHNKE, Bremen, wurden von der Fa. Celler Brunnenbau rd. 60 tiefere maschinelle Bohrungen abgeteuft, die einen guten Einblick in Bau und Verbreitung der quartären Lockerablagerungen verschafft haben. Daraus, auch mittels einer von der erstgenannten Autorin unternommenen pollenanalytischen Untersuchung (gestützt durch von Prof. Mebus Geyh, NLfB, Hannover vorgenommene ${ }^{14} \mathrm{C}$-Datierungen) ist eine relativ verläßliche Rekonstruktion der jüngeren Erdgeschichte möglich.

Die Autoren sagen allen Beteiligten, vor allem aber dem Projektbüro-Leiter von MB-AG, Herrn Dipl.Volkswirt WEDIG vON BELow, herzlichen Dank für die hervorragende Zusammenarbeit und die Erlaubnis zur Veröffentlichung, ebenso den an den Bohrarbei- 


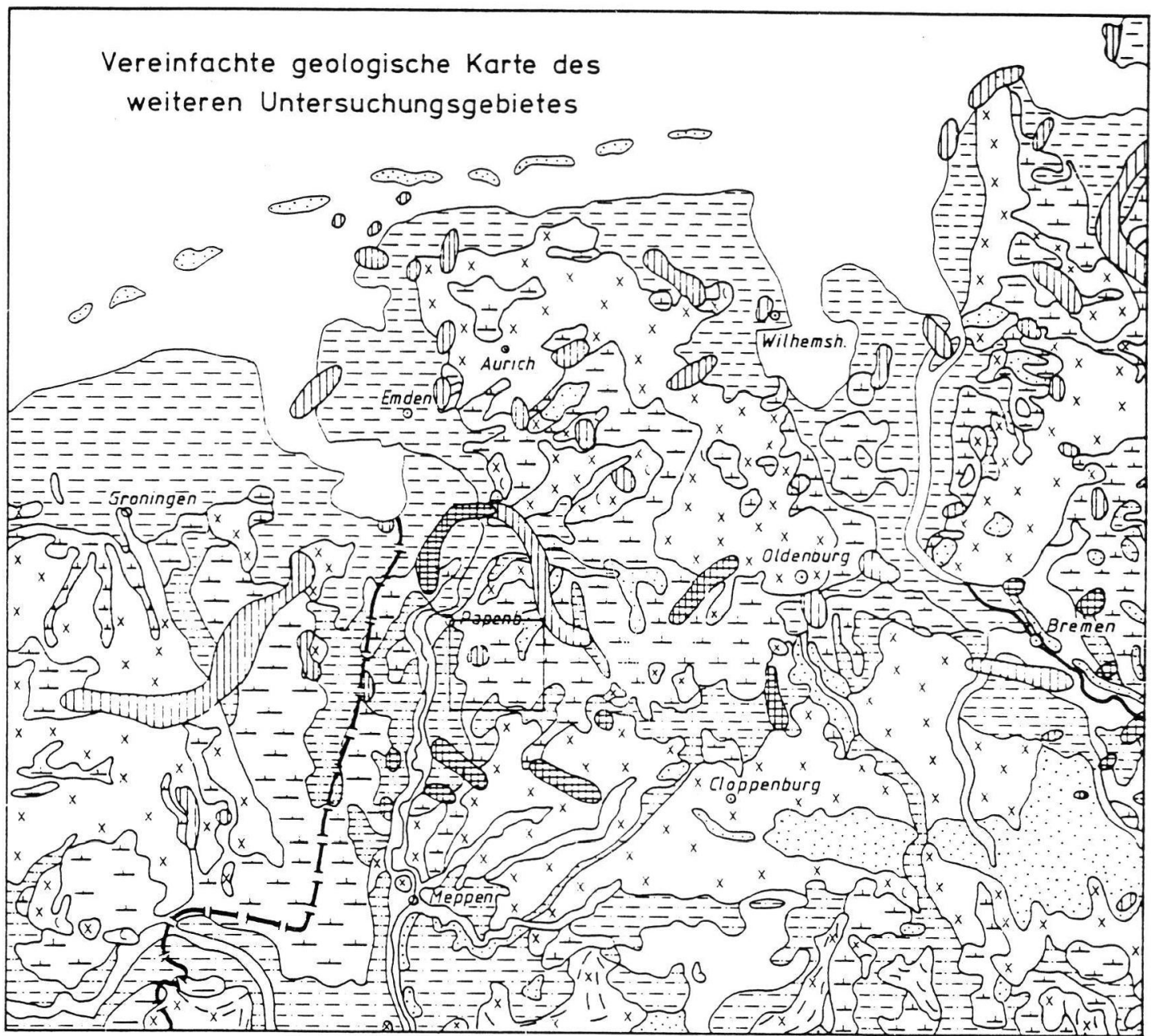

Nach DECHEND \& GRONW.ALD (1962, nach H.-O. GRAHLE.1960) Salzstöcke nach jARITZ (1969)

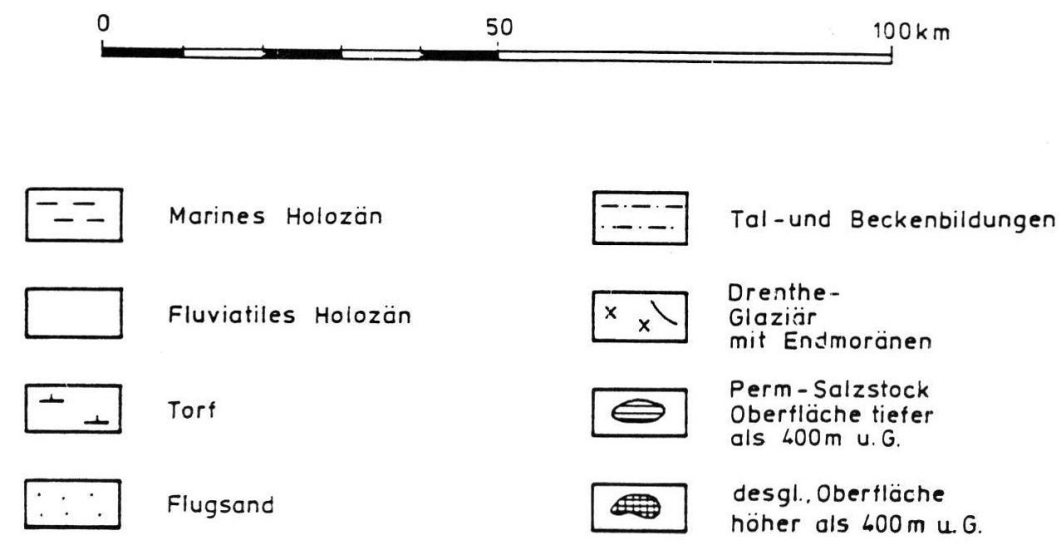

Abb. 1: Stark vereinfachte geologische Karte des Umlandes und des engeren Untersuchungsgebietes (Viereck). 
ten beteiligten Studenten und Diplomanden, v. a. Dipl.-Geol. Horst GAWLIK.

\section{Abriß der Erdgeschichte im Neogen und Pleistozän}

Das engere Untersuchungsgebiet betrifft einen etwa $100 \mathrm{~km}^{2}$ großen Teil des Emslandes, in welchem über den mesozoischen Schichten des Niedersächsischen Tektogens sandige Klastika des Neogens die Basis des Quartärs bilden. Sie bestehen aus Schüttungen aus dem mitteldeutschen Braunkohlenbecken in Richtung auf das neogene Nordseebecken und enthalten lokal Einschaltungen von miozänen Lignit-Flözchen bzw. -Zerreibsel und perimarinen bis marinen Tonlagen im Pliozän.

Diese klastische Folge geht ohne merklichen petrographischen Übergang in altpleistozäne Sande über; darin liegen die Kiese von Neermoor, eine quarzreiche Bildung eines alten Emslaufs. Es ist denkbar, daß diese nach $\mathrm{N}$ gerichtete Schüttung älter ist als die in die altpleistozäne Formation von Enschede gehörende, auch gemischt nordisch-mitteldeutsches Material (Weser-Buntsandstein, Thüringer-Wad-Porphyre, Topas aus dem Weißelstergebiet) enthaltenden östlich-fluviatilen Kiese von Hattem (LÜTTIG \& MaArleveld 1962, K. D. Meyer 1988), die auf eine Schüttung von Osten schließen lassen. Diese wahrscheinlich in die altpleistozäne Menap-Formation der Niederlande zu stellenden Klastika sind Vorboten von Sedimenten elstereiszeitlichen Alters.

Mit den Elster-Sedimenten begannen die eigentlichen Vereisungs-Phasen, das Eupleistozän. Die Elster-Eismassen haben echte Vereisungsspuren aber erst weiter im E hinterlassen. In NW-Deutschland hing der Eisrand dieser Vereisung offensichtlich relativ weit zurück. In den Niederlanden ist kein einwandfreier Nachweis von Elster-Glaziär gelungen.

Im Emsland gibt es mit den Tergaster Kiesen östlich von Emden, nach Bohrergebnissen bei Papenburg (SCHUChT \& TIETZE 1907) und Beobachtungen von K. D. MEYER (1988) in Bohrungen bei Schüttorf glaziäres Elster-Material. Das sind bisher die westlichsten Verbreitungspunkte.

Am Ende der Elstereiszeit gab es in Norddeutschland ein bemerkenswert großes Areal, in welchem als Folge der statischen und dynamischen Exaration unter dem sehr mächtigen Elstereis glaziäre Übertiefungen entstanden, die sich bei Wiederanstieg des Meeresspiegels infolge der damit verbundenen Grundwasserspiegelhebung mit Wasser füllten.

In diesen Becken liegt der dunkelblaugraue, glimmerreiche Lauenburger Ton, der sich nach Westen über die Unterems bis in den Raum von Gro- ningen verfolgen läßt und im Unterweser-UnteremsGebiet Schmink oder Dwo (Schucht 1912), in den Niederlanden pottklei genannt wird. Bei Papenburg stellt er in den meisten Grundwassergewinnungsgebieten eine Aquiclude dar, so daß die darunter liegenden glazifluviatilen Sande der Elstereiszeit bzw. die "präglazialen", weitgehend pliozänen Sande den Förderhorizont abgeben.

Für das nachfolgende Holstein-Interglazial ist eine relativ hohe Erosionsbasis anzunehmen. Im engeren Untersuchungsgebiet sind bisher keine entsprechenden Sedimente gefunden worden. Einer der alten Weserläufe, die in der Zeit Spät-Holstein bis Früh-Drenthe aus dem Gebiete von Minden-Stolzenau bis in die Niederlande verfolgbar sind, läßt sich in der weiteren Umgebung nachweisen.

Im norddeutsch-niederländischen Vereisungsgebiet, v. a. in der Hooge Veluwe, im Raume ItterbeckUelsen und in den Fürstenauer und Dammer Bergen reichen glazifluviatile Kiese und Sande des ältesten Stadiums der Saale-Eiszeit, des DrentheStadiums am weitesten nach Süden. Im Rückland der alten Eisrandlagen sind ausgedehnte Grundmoränenplatten ausgebildet; Drenthe-Geschiebelehm bildet dort das morphologische Rückgrat der Landschaft, so in Hümmling, Oberledinger Land, in der Geest zwischen Emden und Jever.

Bei der Kartierung wurde nirgendwo Geschiebelehm gefunden. Nur an einem Punkt in der Nähe des Hochsitzes im Kortemoor bei re 2600 200, h 5879800 ist eine Geschiebestreuung in Form einer Steinsohle (mit einem großen Findling von rotem Växjö-Granit) im Liegenden des Hochmoor-Torfes aufgeschlossen. Es handelt sich um einen periglaziär umgefrachteten und durch Auswehungsvorgänge zu einer Steinsohle umgearbeiteten Grundmoränenrest über ebenfalls periglaziär umgeformten ehemaligen Becken- und Schmelzwassersanden des Drenthestadiums. Eine Zählung der nordischen Geschiebe in der Steinsohle nach der TGZ-Methode (LÜTTIG 1957) ergab ein theoretisches Geschiebezentrum von 15,7 bis 57,5. Dieser Wert spricht eindeutig für DrentheAlter. Nordisches Kristallin und Klastika erreichen in der Grobkiesfraktion nur $2 \%$, der Rest besteht im wesentlichen aus nordischem Flint (52\%), einheimisch paläozoischen und mesozoischen Quarziten und Sandsteinen (über $20 \%$, Herkunft von Süden), vor allem aber Milchquarz (18\%, wohl im wesentlichen umgelagert aus dem Tertiär). Die liegenden Sande sind eindeutig gut sortierte Beckensande. Es ist anzunehmen, daß sie neben Schmelzwassersanden im Untersuchungsgebiet noch weiter verbreitet sind, als man das bei erster Betrachtung der Bohrproben vermuten kann.

Bei genauer Betrachtung der Bohrproben aus Rammkernbohrungen fällt an mehreren Stellen 
deutlich eine Emersionsfläche auf; die Sedimente darüber und darunter sind schon von der Farbe und Textur her deutlich unterschiedlich.

Aus der äußersten Drenthe-Eisrandlage (Hamelner Phase, LüTTIG 1954) zog sich das Drenthe-Eis staffelartig weit in das skandinavische Heimatgebiet zurück, orientierte sich in dem nachfolgenden Drenthe/Warthe-Interstadial um - das ist statistisch am unterschiedlichen Geschiebeinhalt der Moränen beider Stadien zu sehen (LüTTIG 1960) - und erschien im Warthe-Stadium mit mehr NE-SWlicher Stoßrichtung wieder in Norddeutschland. Dieses Eis hat die (damals erstmalig herausgebildete Unter-) Weser und die Aller nicht mehr überschritten. Falls es daher im Untersuchungsgebiet warthezeitliche Bildungen gibt - sie sind als solche lithologisch-stratigraphisch nicht erkennbar -, könnte es sich nur um periglaziäre, z. B. sandig-lehmige Ablagerungen handeln.

Nach dem Warthestadium kam als nächste Warmzeit das Eem-Interglazial mit der Bildung humoser, wegen des erneuten Meeresspiegelanstiegs im Küstengebiet auch mariner Ablagerungen zur Wirkung. Im Hinterland hat sich das Interglazial im wesentlichen erosiv bemerkbar gemacht. Im Untersuchungsgebiet gibt es zahlreiche Eem-Funde, bis auf das Quakenbrücker Interglazial hat keiner überregionale Bedeutung. Auf das Eem-Interglazial scheint der von Tietze (1907) in einer Bohrung am Zollgebäude in Neu-Rheden in 11 - 13,4 m gefundene Torf hinzudeuten.

Die das Ende des Eem-Interglazials bewirkende Abkühlung beherrschte nicht nur Flora und Fauna, sondern auch das Sedimentationsgeschehen. Über lange Zeit war das Untersuchungsgebiet ein vegetationsarmer, vom periglaziären Klima geprägter Raum mit tiefreichendem Dauerfrostboden, Kongelifraktions-, Kryoturbations- und Solifluktionsvorgängen. Aber auch Windverfrachtung betraf diesen damals trostlosen Raum. Wegen des Dauerfrostbodens war für die Flüsse keine Tiefenerosion möglich, sie waderten, falls ihr Wasser nicht wegen des Frostes stilllag, auf weiten Ebenen daher, im Einklang mit den einebnenden, nivellierenden, gleichmachenden Prozessen der Periglaziär-Abtragung.

Die sich um 25000 B. P. deutlich artikulierenden kaltzeitlichen Vorgänge der Weichsel-E is zeit führten zur Transgression von Eismassen aus Skandinavien bis zur Randlage der Großen Baltischen Endmoräne. Unser Raum blieb weiter Gegenstand der periglaziären Einebnung. In einem von SykeBassum und Uelzen-Wittingen steichenden Streifen kamen auch Flottsande als Vertreter der den Mittelgebirgsraum ummantelnden Löße zur Ablagerung. Bis zum Rückzug des Weichsel-Eises aus der Balti- schen Endmoräne herrschte in unserem Untersuchungsgebiet noch periglaziär-fluviatile Tätigkeit, der wir die Aufschüttung der großen TalsandFlächen im Ems- und Ledagebiet verdanken. Im Grunde genommen ist der Ausdruck inexakt, aber trotzdem treffend. Der Talsandkörper entspricht dem $\mathrm{N}$ i e d e r t e r r a s s e nkörper der anderen norddeutschen Flüsse.

Einige, ältere Autoren bezeichneten dieses Tal als ein altes Urstromtal (Behrmann 1910, Schucht 1913). Diese irreführende Bezeichnung - auf deren Fehlerhaftigkeit auch MEYER \& TüXEN (1986) hinwiesen - ist offensichtlich schwer ausrottbar. Eine Richtigstellung ist notwendig. Der Begriff Urstromtal geht auf die morphogenetische Deutung einer ehemals vereisten Landschaft im Zusammenhang mit der vom Altmeister der Quartärgeologie A. PENcK definierten glaziären Serie zurück (von ihm fälschlich glaziale Serie genannt; "glazial" ist aber ein zeitlicher, nicht ein genetischer Begriff). Danach kann man eine glaziäre Landschaft von innen nach außen wie folgt beschreiben: Innen, d. h. hinter den Endmoränen, liegt die unter dem Eise entstandene (weitgehend aber postglazial geformte) Grundmoränenlandschaft. Die Endmoränen schließen sich als z. T. aufgeschüttete (Satzendmoränen), z. T. gestauchte (Stauchmoränen) Glaziärstrukturen an. Distal zu den Endmoränen liegen die Schmelzwassersande der Sander, in großen Schwemmkegeln aufgeschüttet, ins Vorland immer feinerkörnig werdend. Distal zu den Sandern wird ein großes Talsystem, das Urstromtal angenommen, das die Schmelzwasser aufnimmt und ihren fluviatilen Weitertransport bis zur Vorflut übernimmt.

Nun ist das Ems-Leda-Gebiet, außer von der morphogenetisch vernachlässigbaren Elster-Vereisung, nur von der Drenthe-Vereisung betroffen worden. Von diesem Saale-Stadium kennen wir Endmoränen weiter südlich im Endmoränenzug Itterbeck - Uelsen - Dammer Berge, aber nicht im genannten Gebiet. Es fehlten mithin jegliche Voraussetzungen für die Bildungen eines Urstromtals. Vielmehr ist die als "Urstromtal" bezeichnete Niederung weiter nichts als eine besonders weite Niederterrassenfläche. Sie ist auch erst im Eem-Interglazial vorgeprägt worden und im Weichsel-Glazial entstanden.

Wann die Bildung der Talsande begann, ist unklar. Im Untersuchungsgebiet erregten im Talsandkörper zahlreiche vorhandene und zunächst anscheinend auch niveaugleiche Lignitzerreibsel-Schlufflagen den Verdacht, es könne sich dabei um umgelagertes Eem-Interglazial handeln. Sie sind im Profil der Abb. 2 zu sehen (Höhenlage um +2 bis $5 \mathrm{~m} \mathrm{NN}$ ). Exakte Beweise für diese Vermutung fehlen. Wir wissen aus dem Gebiet westlich der Ems (DÜCKER \& MAARLEvelD 1957, ErBE 1958), daß die Talsandbildung im Weich- 
sel-Spätglazial beendet war, denn zu dieser Zeit legte sich eine ältere äolische "Formation" auf den Körper der Niederterrasse, in den Niederlanden "dekzand" genannt, hierzulande Flugdecksand. In diesen spätglazialen, i. w. noch periglaziären Ablagerungen der Niederlande, Norddeutschlands und Dänemarks, deren Vegetationsbild durch die subarktische Dryas- oder Tundren-Flora gekennzeichnet ist, liegen an vielen Orten nachgewiesene geringmächtige warmzeitliche Schichten, z. T. humos, z. T. als Niedermoortorfe ausgebildet, das Bölling- und AllerödIntervall (11300 - 10600 bzw. 9900 - 9100 v. Chr.). Das Bölling-Intervall ist in den Flugdecksanden der Niederlanden mit der urgeschichtlichen Us selo-Stufe (Hyszeler 1947) ident, von der auch Nachweise im Emsland gelungen sind. Mithin muß die Bildung der Talsande im Spätglazial vor Usselo beendet worden sein. Zur Altersdeutung kann eine von Prof. Geyh, Hannover, im Liegenden der postglazialen (s. Kapitel. 3) Rinne des Burlage-Langholter Tiefs bei re 3402880 h 5880940 in ca. $4 \mathrm{~m}$ NN untersuchte Probe von Holzkohle (Hv 17509) beitragen. Daran wurde ein ${ }^{14}$ C Alter von $29100 \pm 670 \mathrm{v}$. Chr. bestimmt. Das spricht für eine Einstufung in das Denekamp-Thermomer des Weichsel-Pleniglazials.

\section{Die holozäne Entwicklung des Gesamtgebietes}

Das Weichsel-Spätglazial war noch eine Zeit niedrigen Meeresspiegels. Mit der Klimaverbesserung am Ende
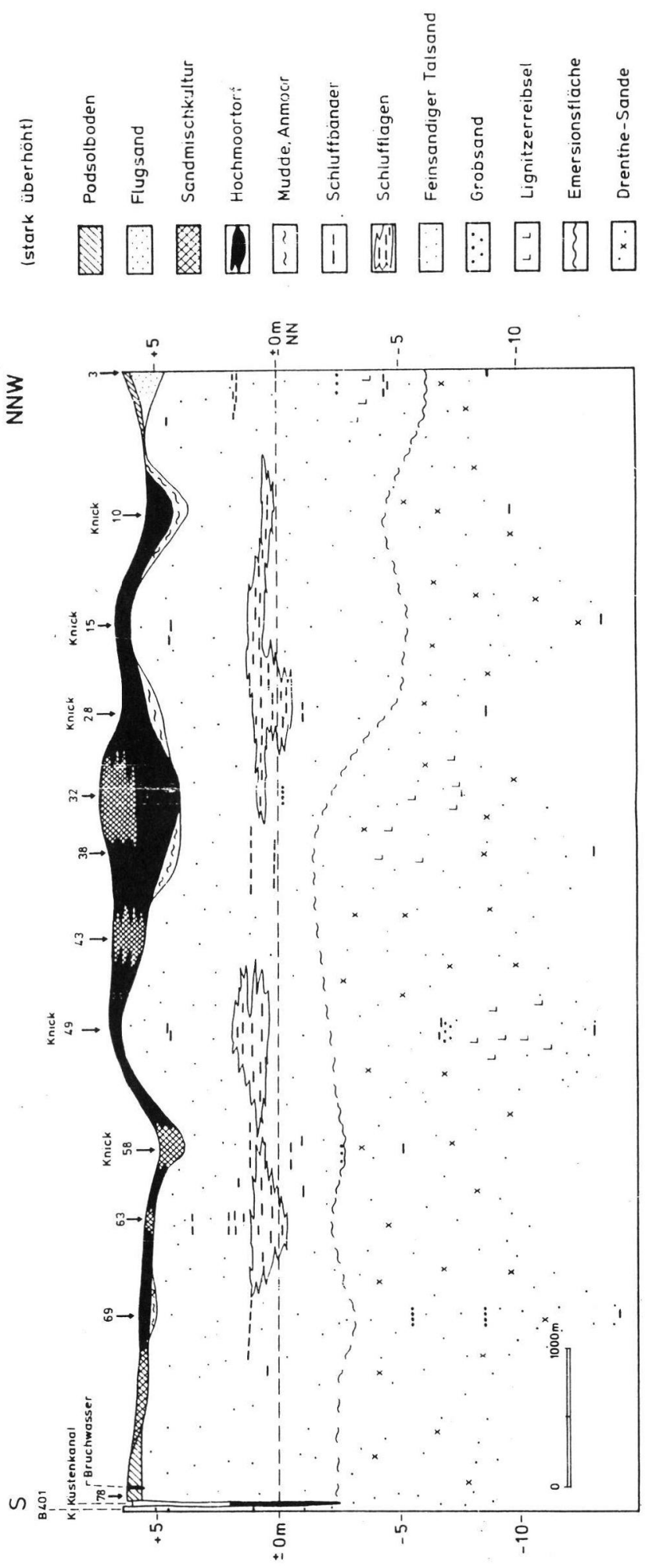

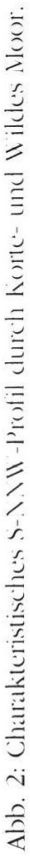




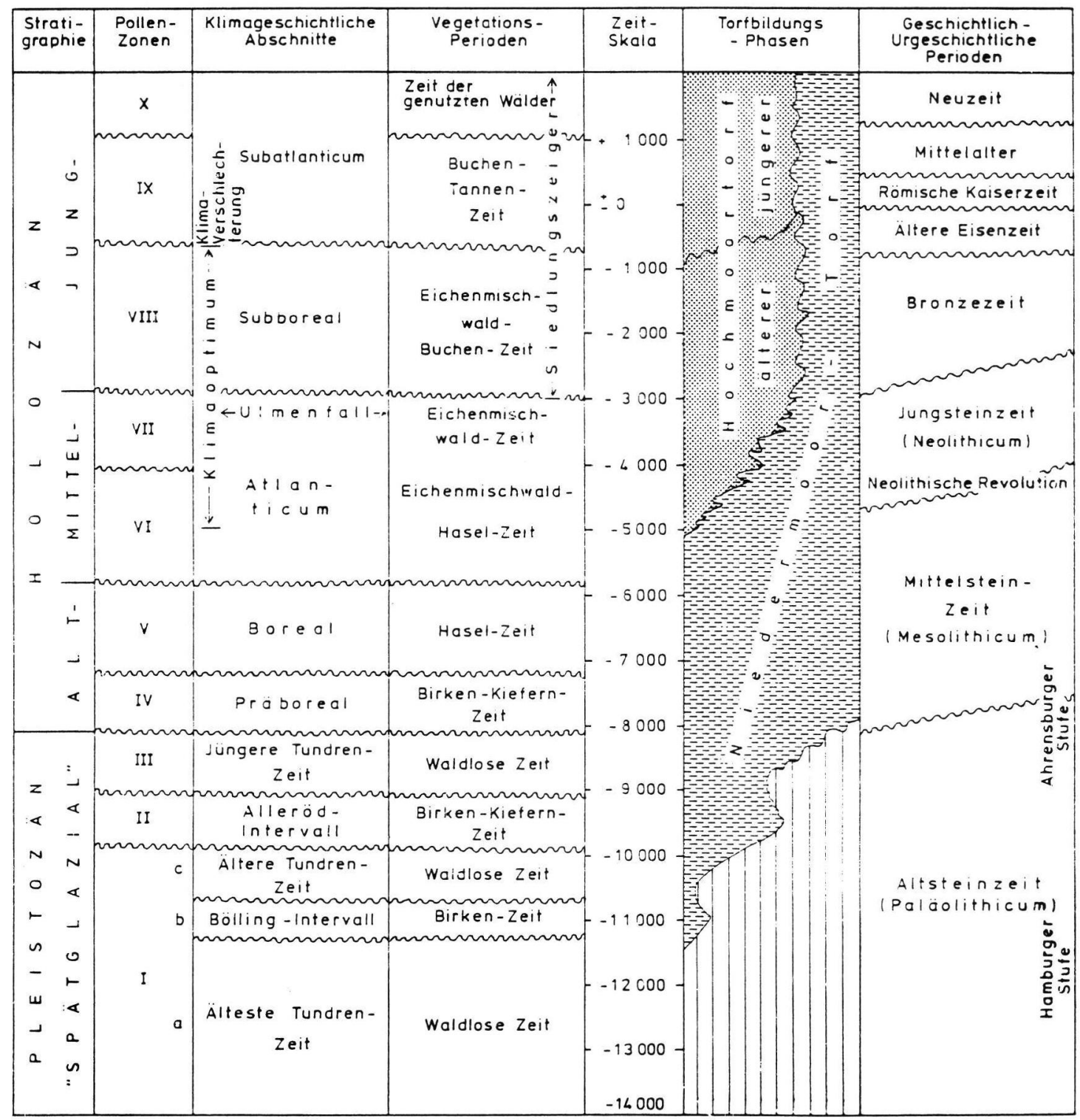

Abb. 3: Pollen- und Klimazonen, Vegetationsperioden, Torfbildungsphasen und urgeschichtliche Abschnitte des Weichsel-Spätglazials und Holozäns.

der jüngeren Dryas-Zeit, an der wir die Grenzen des Pleistozäns zum Holozän ("Postglazial") definieren, verschwand nun auch der Dauerfrostboden, und unsere Flüsse, die der weit in der Nordsee liegenden Küste zuliefen - die Themse war ein linker Nebenfluß, die niederländische Vecht ein rechter Tributär des Rheins -, waren in der Lage, sich entsprechend der tiefen Lage der Vorflut einzuschneiden. Damals müssen die Flußrinnen der Unterems, Leda und z. B. des Burlage-Langholter Tiefs eingeschnitten worden sein. Über die genaue stratigraphische Einstufung dieser Tiefenerosionsphase sind wir bisher nicht hinreichend gut unterrichtet. Es ist an- zunehmen, daß sie i. w. dem Präboreal und Boreal entspricht.

Die holozäne Klimaverbesserung im Atlanticum führte zu einem Wiederanstieg des Meeresspiegels, der allmählich das heutige Küstengebiet erreichte, ja im sogenannten postglazialen Klimaoptimum (ca. 5000 v. Chr.) mit der Flandrischen Transgression diese Linie sogar überschritt. Das Flußgefälle in den Unterläufen wurde dadurch herabgesetzt, die Flüsse zur Aufsandung in den alten Rinnen bewogen und sogar bis in das Ems-Leda-Tal hinein mariner Klei abgelagert. Entsprechend dem erhöhten Grundwasserspiegel wurde auch der Aufwuchs von Nieder- 
moortorf, der schon im Spätglazial begonnen hatte, prononciert. In der Rinne des Burlager-Langholter Tiefs bildete sich in den sandigen Talalluvionen der regional weitverbreitete Tal-Niedermoortorf (TÜXEN 1990). Auf den hochgelegenen Platen begann der Aufwuchs von Hochmoortorf erst später. Sein Wachstums-Maximum erreichte er erst mit der Klimaverschlechterung im Subboreal, v. a. aber im Subatlanticum (Abb. 3).

Vor allem an den Rändern der frühpostglazialen Flußrinnen setzte sich die im Spätglazial begonnene Auswehung der noch nicht vegetationsstabilisierten Talsande fort. Die Flugsand-Akkumulation in Dünenform akzentuierte diese Talränder morphologisch. Das Bild der talrandbegleitenden Flugsande ist nicht nur für das untere Emsland ganz charakteristisch (Pyritz 1972, HAYen 1979, MeYer \& TÜXEN 1986). Dabei ist aber zu berücksichtigen, daß dort auch im Jungholozän Dünen aufgeweht worden sind.

Hinter diesen Talrandbarrieren begann, begünstigt durch bereits altholozäne Podsol-(und damit Ortstein-)Bildung auf den Talsandflächen, in geradezu idealer Position das Hochmoortorf-Wachstum, ausgehend von einer limnisch-telmatischen Phase über Ortsteinen in Schlatts auf der Flugsand-Talsandebene.

Im Zusammenhang mit den Regressionen der Nordsee nach der Flandrischen Transgression wurde die Vorflut zeitweilig wieder tiefer gelegt, und eine komplizierte Ineinanderschachtelung der Erosionsphasen und Akkumulationen ("Decken" im marinen Bereich genannt, Dechend 1956) folgte. Im Tale der Ems und der Nebenflüsse entstand ein tieferliegender Talweg.

Der Talsand trägt in Teilgebieten des Untersuchungsraumes die o. g. Bodenbildung, und zwar einen Podsolboden mit entsprechenden Ortsteinhorizonten, die in der Tat hydraulisch als Aquicluden wirken. Sie greifen auch auf die nächste Einheit, die Flugsande über, soweit es sich dabei um ältere Flugsande (Spätglazial bis Frühpostglazial, K. RicHTER 1951) handelt. Die Podsolbildung ist mithin weitgehend frühholozänen Alters.

Die Flugsande, die, wie bereits bemerkt, vornehmlich der Auswehung von Talsanden im angegebenen Zeitabschnitt entstammen und daher genetisch verknüpft sind mit Ausblasungswannen, bestehen zumeist aus Material, das aus den sehr quarzreichen Talsanden stammt. Die Kornverteilungskurven sind von denen der Talsande nicht sehr gut zu unterscheiden. Das ist ein auch aus anderen Gebieten NW-Deutschlands bekanntes Phänomen: Flugsande sind materialmäßig - übrigens entgegen landläufiger Meinung auch ihre feinerkörnigen Verwandten, die
Löße - sehr eng mit auf das nahegelegene Ausblasungsgebiet bezogen. Wenn das, wie im vorliegenden Falle, eine vegetationsarme Talsandebene war, dann ist es nicht verwunderlich, daß sich petrographische Zusammensetzung wie Kornverteilung der äolischen Sande kaum von der des Ausgangsgesteins abheben. Nur die Lagerungsdichte ist niedriger. Das ist aber ein Faktum, das bei Handbohrungen nicht immer gut zu erkennen ist.

Die bereits genannte jüngere Aufwehungsphase mit einer neuen Dünengeneration steht dann bereits im Zusammenhang mit der menschlichen Einwirkung, ist aber ebenfalls durch die Klimaverschlechterung im Subatlanticum, um 800 v. Chr. beginnend, verursacht.

\section{Die sedentären (i. W. Torfe) einschließlich der limnischtelmatischen Bildungen}

Aus der großen Reihe der Torfe und der sie häufig begleitenden Humit-Sedimente, der Mudden, sind nicht alle Glieder, aber die wichtigsten angetroffen worden.

Die Humitserie baute sich zuerst über den durch die präatlantische (BEIJERNICK 1931, 1933 a, b, FlorSCHÜTZ 1941, HAVINGA 1963) Ortsteinbildung plombierten Ausblasungswannen auf, in welchen eine Versumpfung und subaquatische Akkumulation von Sand-, Ton- und Torfmudden entstand (Versumpfungsmoorbildung). Lokal wuchsen über diesen Mudden Niedermoor- und/oder Bruchwaldtorf auf.

Dieser Versumpfungsmoor-Typ, während dessen Bildung lokal und zeitweise telmatische Bedingungen herrschten, erfaßte vornehmlich die Schlatts und war eine Vorlaufphase zur ombrogenen Hochmoortorf-Phase, deren Torfe sich gebietsweise über den Versumpfungsmoor-Zyklus aufbauten, in anderen Arealen aber direkt auf den Podsolen des Talsandes oder der älteren Flugsande wurzelecht aufwuchsen. Ein charakteristisches N-S-Profil durch die HumitLagerstätten ist in Abb. 4 wiedergegeben.

$\mathrm{Zu}$ beachten ist, daß der Hochmoortorfaufwuchs (älterer Hochmoortorf), der schließlich zu einem einheitlichen Moorgebiet führte, im Untersuchungsraum in den drei unterschiedlichen Zentren, dem Wilden Moor (südlich von Papenburg), dem Kortenmoor (östlich des Wilden Moores) und dem Klostermoor (NW von Neuburlage) entstand.

Aus diesen drei Nuclei wuchsen, während der Bildungs-Zeit des älteren Hochmoortorfs (Schwarztor- 
fes) beginnend, aber in Vollendung mit dem jüngeren Hochmoortorf (Weißtorf), die drei entsprechenden Hochmoortorf-Gewölbe (sie sind als getrennte Einheiten an den Höhenlinien der topographischen Landesaufnahme des letzten Jahrhunderts noch zu erkennen) zu einem geschlossenen Moor zusammen. Es handelt sich hier mithin um den Urtyp des "gewölbten Hochmoores" (JonAs 1934, 1935) mit Randgehängen und Laggs (= Moorrandkolke), nicht um den am Nordhümmling vorhandenen Heidemoortyp, den EgGeLsMANN \& BlankENBURg (1990) beschrieben haben.

Der zwischen dem (stark zersetzten) Schwarztorf und dem (weniger gut zersetzten) Weißtorf in den norddeutschen Mooren gewöhnlich beobachtete Grenzhorizont, eine Emersionsbildung, die man früher als synchron betrachtet hat - was aber nicht richtig ist, wenngleich von einer Periode gesprochen werden kann, in welcher solche von Ort zu Ort gering zeitverschiedene Marken sich häuften -, tritt in den Papenburger Mooren nur sehr lokal in Erscheinung. $\mathrm{Zu}$ bedenken ist aber, daß wir uns in einem Abtorfungsgebiet bzw. einem durch Kultivierungsmaßnahmen veränderten Moorgebiet befinden, in dem der Weißtorf bereits weitgehend abgebaut oder umgebrochen worden ist.

Verbreitungsmäßig tritt aus den ansonsten vorhandenen, wegen ihrer geringen Bedeutung hier nicht abgehandelten Torfvarietäten noch der in vielen Profilen an der Basis des Schwarztorfes verbreitete Eriophorum-vaginatum-Torf auf (scheidiges Wollgras, Moorbart, im Emsland "Speckrücken" genannt). Die Torfgräber pflegten früher Ausgang des Winters die unterirdischen Stengelteile zu verzehren (SCHLICHT 1954).

Der hangende Weißtorf ist bereits weitgehend der Torfgewinnung anheimgefallen oder bei der Kultivierung verändert worden.

Im Ostteil des Kortemoores ist in Neuburlage westlich des Burlage-Langholter Tiefs am Westrand des hier verbreiteten, auf der Talsandfläche sitzenden Jungdünengürtels ein den nòch erkennbaren östlichen Randgehänge des Moores begleitendes Lagg auskartiert worden. In einem zeitweilig offenen Aufschluß war erkennbar, daß sich im Laggbereich von Randgehängen abgeschwemmter (oder abgewehter) Torf mit von den Dünen abgeschwemmtem Flugsand in feiner Wechsellagerung vermengt (Abb. 5).

Hingewiesen werden muß noch auf die Tatsache, daß vor der Abtorfung und vorausgehenden Trockenlegung noch einzelne Hochmoorkolke vorhanden waren, die inzwischen blind geworden sind, so v. a. das in bezug auf seine Vegetation interessante Brunselmeer.

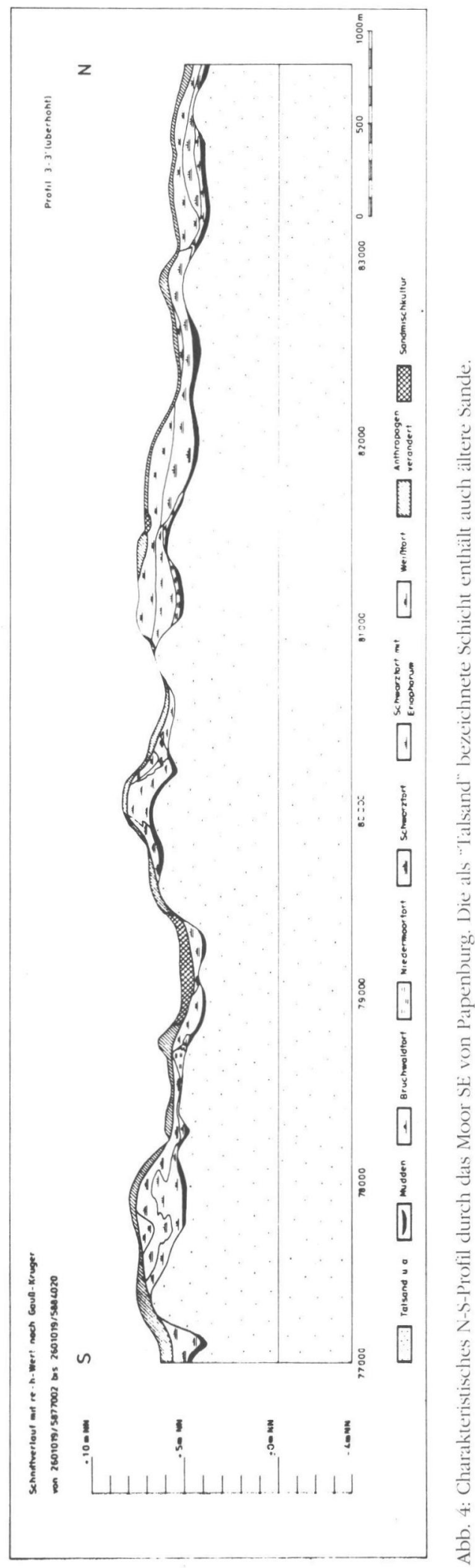




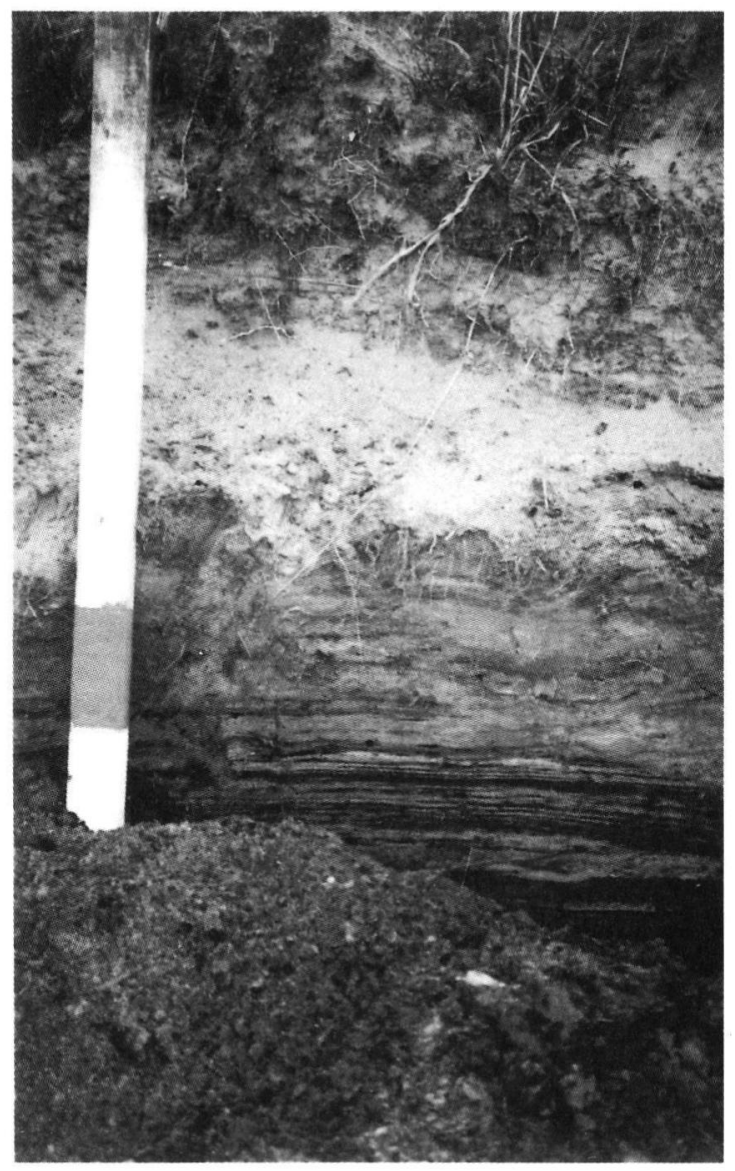

$\begin{array}{ll}0,14-0,17 \mathrm{~m}, & (\mathrm{Nr} . \mathrm{Hv} 17270)+445 \mathrm{n} \mathrm{Chr} \pm 70 \\ 1,09-1,13 \mathrm{~m}, & \text { (Nr. Hv 17 271) }-435 \pm 70 \\ 2,10-2,14 \mathrm{~m} \text { und } & (\mathrm{Nr} . \mathrm{Hv} 17272)-1685 \pm 75 \mathrm{v} . \mathrm{Chr} . \\ 3,00-3,03 \mathrm{~m} & \text { (Nr. Hv 17 273) }-3270 \pm 75 \mathrm{v} . \text { Chr. }\end{array}$

Auf die für die Vegetationsgeschichte wichtige Untersuchung von F. JonAs (1956) im nördlich benachbarten Klostermoor muß hingewiesen werden. Angemerkt werden muß aber, daß bei den Daten von JoNAs schwer zwischen Dichtung und Wahrheit unterschieden werden kann (H. MülLLER 1956).

Im einzelnen ergibt sich bezüglich der Vegetationsgeschichte nach dem Pollendiagramm und der vorliegenden Literatur (OVERBECK \& Schmitz 1931, JoNAS 1956, H. MÜLLER

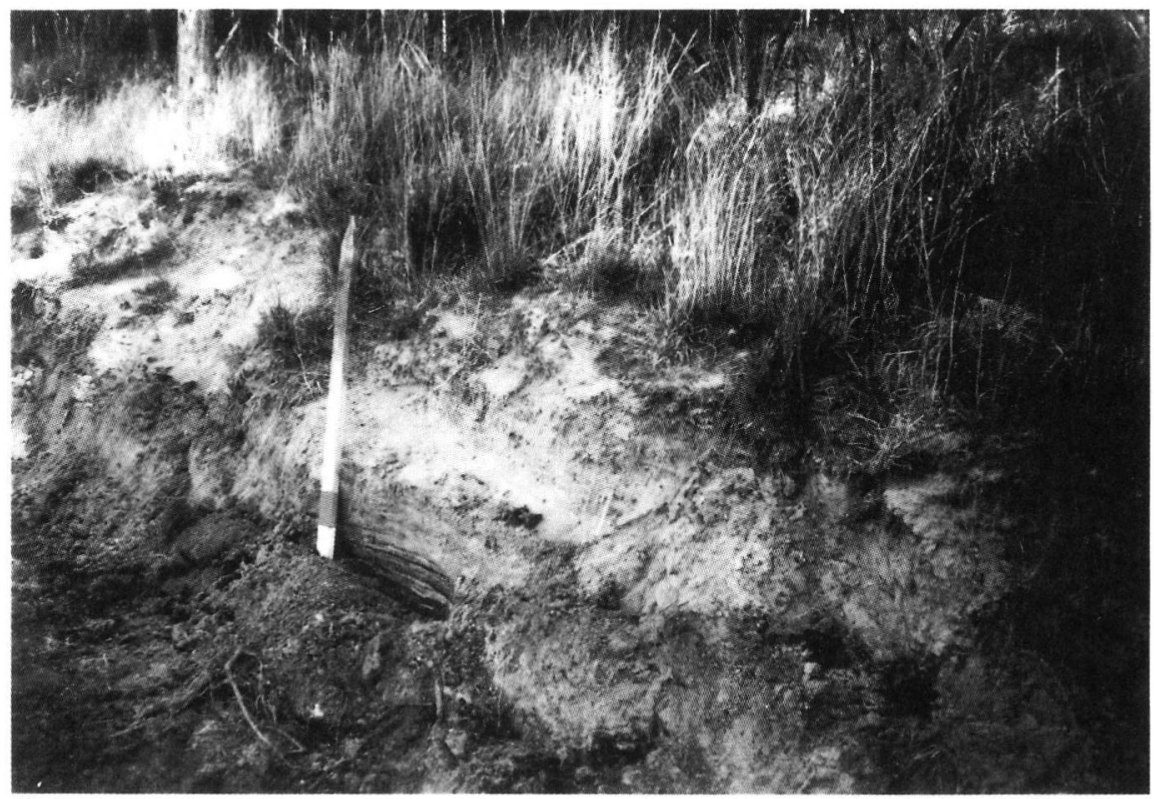

Abb. 5: Feine Wechsellagerung von in Lisgalureich abgeschwemmtem (oder verwehtem) Hochmoortorf mit von der benachbarten jungholozänen Düne bei Neuburlage abgeschwemmtem Flugsand. Pkt. re 3402 880, h 5882 525, Blatt Burlage TK 25, Nr. 2911, Okt. 1990.
1956, BEUG 1957, KUBITZKI 1961, BEHRE $1970, \quad 1985$, KRAMM 1978, 1981, VAN GEEL 1978, ISENBERG 1979, DuPONT \& BRENNINKMEIJER 1984, MiDDELDORP 1984, DuPONT 1986) folgendes:

Die im Spätglazial verbreitete Tundrenvegetation wurde in den zwei relativ kurzen Thermomeren, dem B ölling - und A lle r ö d-Intervall, vorübergehend durch Baumbewuchs, bei welchem v. a. die Moorbirke, dane- 
ben die Kiefer dominant gewesen sein mögen, unterbrochen. In der jüngeren Tundrenzeit (Pollenzone III nach FIBRAS) bildete sich erneut eine vegetationsarme, infolge periglaziärer und äolischer Vorgänge unwirtliche Landschaft heraus.

Mit dem endgültigen Temperaturanstieg zu Beginn des Holozäns, im Präboreal (Pollenzone IV), ergriff die Baumvegetation von dieser Landschaft Besitz. Zuerst war es die Birke, dann die Kiefer, bis im Boreal die Erle, zögerlich auch die Hasel zur Entstehung lichter Wälder führten (Pollenzone V, BirkenKiefern-Hasel-Zeit). Auch die ersten Abkömmlinge des Eichenmischwaldes sind zu verzeichnen. In unserem Torfprofil ist diese Vegetationsperiode durch die beginnende Versumpfung gekennzeichnet. Auf einem geringmächtigen Moorerdehorizont in einer Senke im Talsand folgte die Bildung einer sandigen Mudde. Diese gehört in ihrem oberen Teil auch noch in die Zeit der massenhaften Erlenausbreitung und in das frühere Atlanticum und zugleich in das ausgehende Mesolithicum. Die Eichenmischwaldvegetation ist durch ein Maximum der Ulmenpollen gekennzeichnet, gleichzeitig ging die Kiefer zurück (Pollenzone VI). Im Torfprofil sind noch immer limnisch-telmatische Bedingungen zu erkennen.

Pollenzone VII, das jüngere Atlanticum, zeigt eine stabile Eichenmischwald-Gesellschaft. Die Beteiligung der Hasel ging unter $25 \%$ zurück. Im Torfprofil ist Niedermoortorf repräsentiert; erst in der zweiten Hälfte der Pollenzone VII begann die Hochmoortorf-Bildung, gleichzeitig mit der endgültigen Besiedlung (während der Neolithischen Revolution). Es muß festgehalten werden, daß es zur Zeit des Seßhaftwerdens der mesolithischen Populationen noch kein Hochmoortorfwachstum gab. Der aufwachsende Schwarztorf war gleichzeitig ein Eriophorum-Sphagnum-Torf. Dieser Reichtum des Schwarztorfes an Eriophorum ist eine Tatsache, auf die bereits Overbeck \& Schmitz (1931) hingewiesen haben. Schwarztorf und Weißtorf sind eben zwei litho- und biofaziell völlig unterschiedliche Torfarten, und es liegt an diesem Unterschied, daß der Schwarztorf wesentlich besser zur Humifizierung neigte als der Weißtorf. Charakteristisch ist auch, daß im untersuchten Profil ein typischer Versumpfungsvorgang überliefert ist. Wir befinden uns in einem Altmoränengebiet, in welchem größere Wasserbecken, wie sie im Jungmoränengebiet zu limnisch-telmatischen Bedingungen führen konnten, fehlten (OvERBECK \& SCHMITZ 1931).

Pollenzone VIII, das Subboreal, normalerweise in Nordwestdeutschland vegetationskundlich eine Eichenmischwald-Buchenzeit, ist in unserem Torfprofil im Schwarztorf, von der Mitte ab aufwärts ohne Eriophorum, enthalten. Die Hasel ist wieder mit mehr als $40 \%$ im Pollendiagramm vertreten. Ent- sprechend der neolithischen Besiedlung sind siedlungsanzeigende Pollen zunehmend vertreten, v. a. Plantago lanceolata schon vom unteren Teil ab. Die Nichtbaumpollenwerte nehmen deutlich zu.

Die Grenze von Pollenzone VIII/IX, der Übergang vom Subboreal zum Subatlanticum, ist durch zurückgehende Buchenpollenwerte charakterisiert. Um 700 v. Chr. ist ein Haselpollenrückgang auf unter $10 \%$ deutlich. Das ältere Subatlanticum, Pollenzone IX, begann bekanntermaßen mit einer Klimaveränderung vom trockenen und wärmeren Subboreal zum feuchten und kühleren Älteren Subatlanticum. Hainbuchen- und Buchenkurven steigen deutlich an. Diese Pollenzonengrenze liegt noch in der Periode der Schwarztorfbildung, der Umschlag zum Weißtorf fand erst in der Mitte der Pollenzone IX (vermutlich um Christi Geburt) statt. Das ist ein vergleichsweise junger Beginn der Weißtorfbildung. An Weißtorfarten sind, wie im westlichen Niedersachsen üblich (Tüxen 1990), Sphagnum imbricatum, magellanicum und papillosum wesentlich vertreten, aber auch acutifolium, fuscum und cuspidatum kommen vor.

Das Profil endet (wegen des durch den Abbau bedingten Fehlens der Pollenzone X) bereits vor dem frühen Mittelalter (Jüngeres Subatlanticum). Der für diese Zeit typische Anstieg der Siedlungsanzeiger, vornehmlich von Secale, wurde nicht mehr festgestellt. Bei der im Pollendiagramm überlieferten Depression in der Kurve der Siedlungsanzeiger in 15 cm Teufe dürfte es sich um die Auswirkung der Völkerwanderungszeit handeln. Dieser Rückgang, der von der Zunahme der Buche und der Birke begleitet wird, wird ins 4. Jahrhundert n. Chr. datiert.

\section{Menschliche Einflüsse auf das erdgeschichtliche Geschehen}

Während sich auf den höher gelegenen Geestinseln, wie dem Hümmling, die urgeschichtliche Besiedlung bis ins Paläolithikum zurückverfolgen läßt (SCHLicht 1954), ist auf den tieferen Landesteilen der Mensch erst relativ spät seßhaft geworden. Das wird auch verständlich, wenn man bedenkt, daß die Talsandebene sich noch während des späten Paläolithikums entwickelte und daß die Überdeckung durch die älteren Flugsande erst begann. So finden sich auch erst nach Bildung der älteren Dünengeneration menschliche Hinterlassenschaften v. a. am Rande der Flußtäler der Ems und des Burlage-Langholter Tiefs. Aber auch die mesolithischen und neolithischen Spuren sind dort recht dürftig. Das Unteremstal war im Neolithicum außerordentlich schwach besiedelt, ein Zustand, der sich bis in die Bronzezeit fortsetzte (KotTMann 1963). 
In den Mooren südöstlich von Papenburg sind Siedlungsspuren außerordentlich selten (vgl. auch SCHLÜTERs 1986, eindrucksvolle Karten). An höheren, aus dem Torf heraufragenden Stellen, wie an der Düne am Vosseberg (JonAS 1941 a) sind bronzezeitliche Funde gemacht worden. Rink (1935) meldete neolithische Feuersteinfunde vom Rande des Langholter Tiefs aus Altburlage, einen Eichbaumkahn unbekannten Alters vom Langholter Tief und einen Bronzedepotfund aus dem Moor von Ostrhauderfehn.

Bemerkenswert ist auch das (bisherige) Fehlen von Bohlwegen in den Papenburger Mooren (frdlm. mündl. Mitteilung von Dr. h. c. Hajo Hayen). Nur ein Hinweis von JoNAS (1941 a) existiert, nach welchem die in der Nähe des Vosseberges wohnenden Einheimischen seinerzeit noch von der Existenz eines alten Bohlweges gewußt haben sollen.

Die obgleich schwache bronze- und eisenzeitliche Besiedlung deutet sich durch die Siedlungsanzeiger im Pollendiagramm (Beil. 1) an. Nach Jonas (1941 a) ist im 2. vorchristlichen Jahrtausend mit Getreideanbau zu rechnen. Seine Angabe über einen Rückgang des Getreideanbaus mit Beginn der Pollenzone IX scheint verläßlich zu sein, ebenso wie der Hinweis auf einen erneuten Anstieg in der zweiten Hälfte des 1. vorchristlichen Jahrtausends. Um die Zeitenwende war das Gebiet von den Ampsivariern besiedelt, die 59 v. Chr. von den Chauken verdrängt wurden. Die Besiedlung konzentrierte sich, wie bereits erwähnt wurde, an den Rändern der Flußtäler.

Zur Zeit der Völkerwanderung sind die Spuren des Ackerbaues wiederum dürftig, ebenso um $700 \mathrm{n}$. Chr. in der sächsischen Rodungsperiode.

Man muß damit rechnen, daß die Plaggenwirtschaft zum Zwecke der Düngung der kargen Böden nicht, wie KotTMAnn (1963) vermutete, schon um $400 \mathrm{n}$. Chr., oder wie FASTABEND \& V. RAUPACH (1962) angaben, schon um 700 v. Chr. einsetzte - das erscheint bereits aufgrund der schwachen Besiedlung in der vorsächsischen Zeit unwahrscheinlich -, sondern erst um 950 n. Chr. (BeHre 1976) begann. Diese Zeit entspricht auch dem Beginn des Roggenanbaues in unserem Gebiet.

Entgegen der allgemein verbreiteten Annahme, der Buchweizenanbau habe erst mit der Kreuzfahrerzeit begonnen, vermutete JonAs (1941 a), daß der Beginn um $1200 \mathrm{n}$. Chr. gleichzeitig mit dem von ihm postulierten 4. Getreidegipfel- läge. Danach sei ein landeskultureller Niedergang um $1250-1300$ n. Chr. und um 1400 n. Chr. als Folge der Fehde des Ravensburger Geschlechts mit den Nachbarn (sog. Raubritterzeit) bzw. des Aschendorfer Bauernaufstandes im Emsland zu bemerken. Der letzte Einschnitt entspricht den Glaubenskriegen. Bei der
Neuinstallation des 1379 gegründeten Papenburgs im Jahre 1630 bzw. 1638 - GRAF vON VELEN mußte zwischendurch wegen der schwedischen Besetzung fliehen - wurde von einem wüsten Gebiet gesprochen, in welchem die Schaftrift zahlreiche Sandverwehungen hervorgerufen habe (A. HugenBerg 1891). Dieser vor allem südlich von Papenburg verbreiteten Erscheinung ist erstmals ein Bürger MEYER aus Papenburg mit Aufforstungsmaßnahmen entgegengetreten, auf welche die Flurbezeichnung MEYERs Tannen zurückgeht.

Mit der Papenburger Gründungszeit treten wir gleichzeitig in die Geschichte der Kultivierung der emsländischen und ostfriesischen Moorgebiete ein.

Die von Holland übernommene Fehnkultur, die in erster Linie die "Kolonisation" siedlungsarmer Gebiete zum Ziele hatte und im Bourtanger Moor 1599 erstmals auf deutschen Boden übergriff, hatte, vereinfacht gesagt, zum Ziel, den Torf abzugraben, um dann den Untergrund zu kultivieren (cultivare = pflegen; diese Begriffsbestimmung scheint einigen Zeitgenossen unbekannt zu sein!). Zur zügigen Entwicklung des entsprechenden Landes war die Anlage schiffbarer Kanäle notwendig, um einerseits den Brenntorf zur Kundschaft zu befördern, andererseits den erforderlichen Dünger anzufahren (FreEse 1789, ARENDS 1820, DyCKerhoff 1927, A. Wegener 1985).

Damit erfolgte der erste großmaßstäbliche landeskulturelle Eingriff in die nordwestdeutschen Hochmoore, während Nieder- und Bruchwaldtorfgebiete schon im 11. bis 13. Jahrhundert, besonders durch die Tätigkeit der Zisterzienser, nach einer Zeitlücke dann im 17./18. Jahrhundert durch die Landesherrn kultiviert worden waren. Mit der ersten Tätigkeit der Mönche in Zusammenhang ist auch die Gründung der Klöster Osterholz (1182) und Lilienthal (1232) im Bremischen zu sehen (A. HugEnBERG 1891).

Basis der Fehnkultur ist eine systematische Entwässerung über die "großen Arterien eines Fehnorganismus", die Kanäle, die aus einem ganzen System von Stammkanälen (Wieken) und Seitenkanälen (Inwieken) bestehen. Von letzteren zweigten dann noch Achterwieken ab, die direkt zu den Torfstichen führten. Die Häuser der Kolonisatoren wurden zunächst direkt auf den Torf, später auf das abgetorfte Gebiet, das Leegmoor gebaut. Regelmäßig lange, ca. 10 ha große Grundstücke, die Moorhufen, wurden an die Siedler vergeben. 1633 wurde die erste Fehnkultur mit der Gründung des Großefehns in Ostfriesland und, wie gesagt, 1638 die Fehnkolonie Papenburg angelegt.

Mit der Verfehnung ging das Moorbrennen einher; nach Ostfriesland wurde es 1707 von Pastor BOLENIUS aus Holland übertragen. 
Als die Preußen im Jahre 1744 Ostfriesland erwarben, ging ihnen die Fehnkultur zu langsam, und sie entwickelten auf der Basis der Moorbrandkultur die Buchweizenkolonien, wobei man mit der Besiedlung meist vom Geest/Moor-Rand ausging. Das ging in Ostfriesland zunächst gut, bis durch das ständige Brennen eine Nährstoffverarmung eintrat. Man muß davon ausgehen, daß nach 6- bis 10jährigem Brennen das Moorgebiet 15 - 30 Jahre liegen gelassen werden mußte, damit sich die Bodenkrume erholen konnte. Auch das ungeschickte Pachtsystem war daran schuld, daß ein allmählicher Verfall eintrat. Ähnliches geschah in den hannöverschen und oldenburgischen Gebieten. Um 1840 waren die Moore weitgehend ausgebrannt, und die Rettung kam erst um 1870 mit dem Mineraldünger.

Die Kolonisation im Emsland und in den Herzogtümern Bremen und Verden geschah für die Siedler in günstiger Weise. In den Münsterschen Landen wurde die Moorkolonisation nur aus politischen Gründen, und zwar, um die Grenze gegen Holland durch Besiedlung zu schützen, vorgenommen (A. HugeNBERG, 1891). Die Landesherren waren aber damit wenig erfolgreich, auch die Bentheimer Moorbrandkolonisation. Einzige relativ erfolgreiche Fehnkolonie war Papenburg, und auch in dem Arenberger Moorgebiet - so wurden die Moore SE von Papenburg nach A. Hugenberg früher genannt - brachte erst die Mineraldüngung den eigentlichen Erfolg. Insgesamt war der kultivierte Flächenanteil aber gering. Preußen, das 186636800 ha Moorland im Emsland erworben hatte, hat bis 1949 davon nur 9400 ha kultiviert (G. Hugenderg 1973).

Beim Kanalbau im Raume Papenburg machten die bereits erwähnten Ortsteinlagen große Probleme, zumal bei der Ausweitung des Kanalnetzes nach Osten (JoNAS 1938 a). So mußte der ursprüngliche Plan, vom Unterende geradewegs nach Osten zu graben, aufgegeben werden. Statt dessen wurde der Durchstich durch das Ortsteingebiet an eine schmalere Stelle nordwestlich verlegt, und der ursprüngliche erste Kanal, der jetzige Gasthauskanal, blieb ein Torso. Die Papenburger Moorsiedler mieden überhaupt wegen des Ortsteins die Sandgebiete, die sie wegen ihrer ortsteinbedingten Waldfeindlichkeit Urheide nannten.

Auch hier brachten erst der Einsatz von Mineraldünger mit der Deutschen-Hochmoor-Kultur, vor allem aber die mit Tiefpflugmaßnahmen verknüpfte Sandmischkultur die positive Wende.

Es ist ein Irrtum zu glauben, die erste Kolonisationsphase habe trotz des Abbrennens der oberen Weißtorfschichten, des Buchweizenanbaus und der Schafbeweidung den Naturraum wesentlich und tiefgreifend verändert. Dafür verantwortlich sind nicht die Kolonisationsmaßnahmen des 17. und 18. und der ersten Hälfte des 19. Jahrhunderts. Die große Moor-Aufzehrung geschah erst später. Man kann das sehr gut an den Veränderungen des Bildes der topographischen Karten sehen.

Zur Verfügung stehen dazu Karten der Landesaufnahme mit verschiedenen Aufnahmejahren, beginnend mit der Gaussschen Landschaftsaufnahme von $1860 / 61$ bis 1987 .

Nimmt man die in den Jahren 1860/61 festgestellte Moorfläche als Ausgangsfläche, was sicherlich nicht ganz richtig ist, gleich $100 \%$ Fläche, so ist gemäß Abb. 6 zu erkennen, daß die entscheidenden Veränderungen in der Zeit des Dritten Reiches - wir wollen die entsprechenden wenig erfreulichen Akte, die dazu führten, nicht im einzelnen erörtern - sowie kurz nach dem 2. Weltkrieg (Emslandplan, Küstenplan) geschehen. Man muß dazu bedenken, daß im Emslande noch im Jahre 194952 \% der Erwerbspersonen in der Landwirtschaft tätig waren (FRANKE 1982). Dabei ist die Tätigkeit der am 1. Januar 1950 im Bundestag beschlossenen Emsland $\mathrm{GmbH}$ und ihres Geschäftsführers J. D. Lauenstein als eine der größten landeskulturellen Leistungen in der Nachkriegszeit besonders herauszuheben. Es ist auch notwendig, ausdrücklich zu bemerken, welche enorme Bedeutung diese landeskulturellen Maßsnahmen angesichts der Nachkriegs-Ernährungs- und Flüchtlingsprobleme besaßen. 1965 waren die entsprechenden Siedlungsmaßnahmen abgeschlossen (G. Hugenberg 1973).

Der von 1912 ab geplante, 1926 begonnene Bau des Küstenkanals hat zweifellos auch indirekt den Flächenschwund der Moorgebiete begünstigt.

\section{Schlußwort}

Das entscheinde Faktum für das MB-AG-Projekt ist in diesem Zusammenhang folgendes: Die geplanten Baumaßnahmen betreffen ein Gebiet, welches nur mehr ein blasser Abglanz einer natürlichen Landschaft ist. Daraus ist zu folgern, daß Vorsicht gegen Ansprüche, diesen Landschaftsteil als urtümlich, naturnah und daher schützenswert zu bezeichnen, angebracht ist. Aus diesem Grunde erscheint es als besonders bemerkenswert, daß MB-AG keine Anstrengungen unterlassen hat, bereits in der Phase der Voruntersuchung alle dem Naturschutz zugute kommenden Forschungen für die Feststellung des Biotop-Ist-Zustandes unter Einsatz erheblicher Mittel zu betreiben. Des weiteren ist es das erklärte Ziel, mit den notwendigen Baumaßnahmen Ausgleichsleistungen für eine naturnahe Gestaltung der Innenund Außenflächen des Prüfgeländes, für dessen Anlage inzwischen die generelle Zustimmung der Nie- 


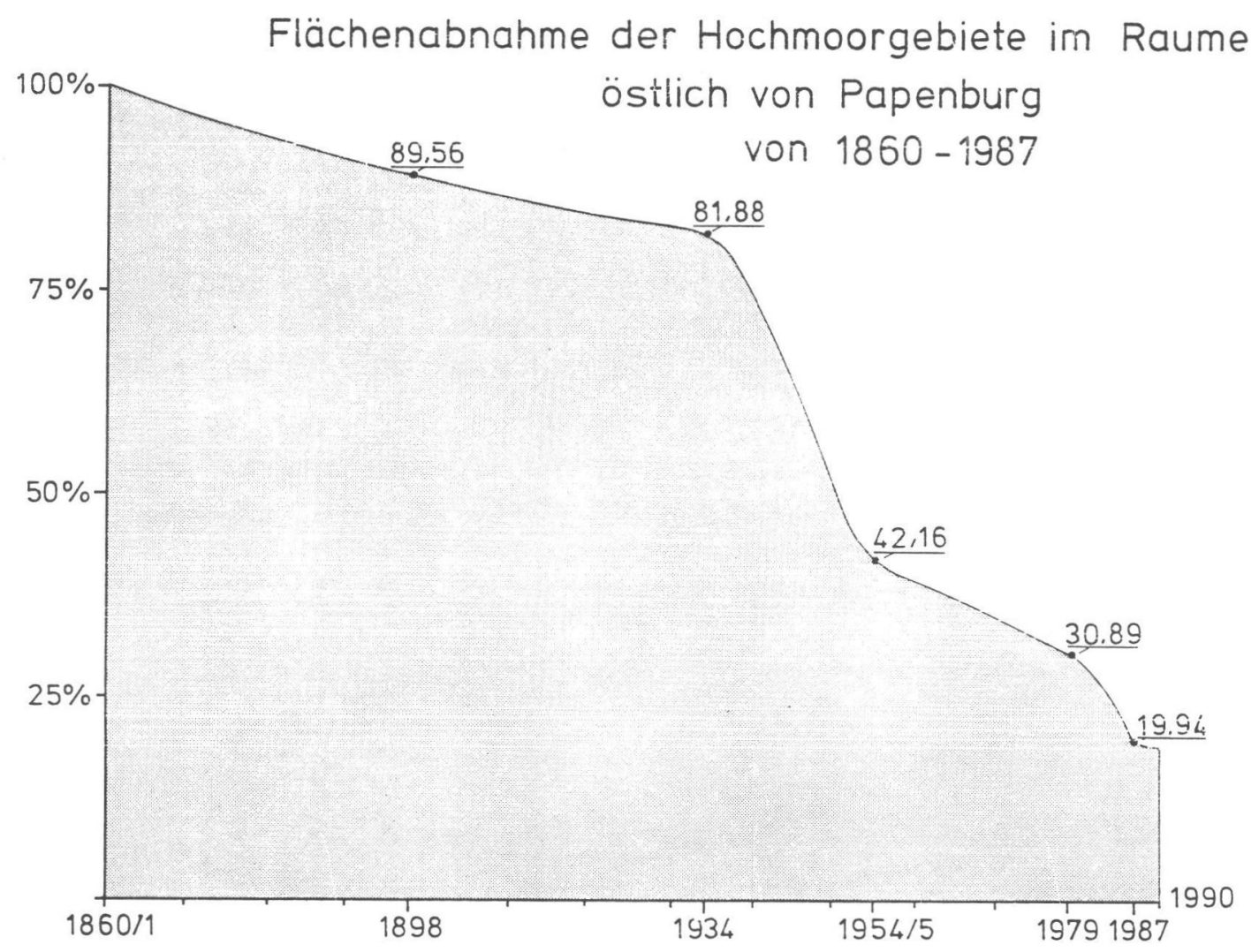

Abb. 6: Flächenabnahme der Hochmoorgebiete im Raume östlich von Papenburg von 1860 bis 1987.

dersächsischen Landesregierung vorliegt, zu verbinden.

\section{Schriftenverzeichnis}

ARENDS Friedrich (1820): Die Fehne. - Ostfriesland und Jever in geographischer, statistischer und besonders landwirtschaftlicher Hinsicht. - 3: 486 - 501, Hannover (Hahn) 1820.

BeHre, Karl-Ernst (1970): Die Entwicklungsgeschichte der natürlichen Vegetation im Gebiet der unteren Ems und ihre Abhängigkeit von den Bewegungen des Meeresspiegels. - Probl. d. Küstenforsch. südl. Nordseegeb. 9: 13 - 47, 10 Abb., 7 Tab., Hildesheim 1970.

- (1976): Beginn und Form der Plaggenwirtschaft in Nordwestdeutschland nach pollenanalytischen Untersuchungen in Ostfriesland. - N. Ausgrab. Forsch. Nds. 10: 197 - 224, Taf. 7 - 10, 6 Abb., 6 Tab., Hildesheim 1976.

- (1985): Die ursprüngliche Vegetation in den deutschen Marschgebieten und deren Veränderung durch prähistorische Besiedlung und Meeresspiegelbewegungen. - Verh. Ges. Ökol. 13: 85 - 96, 6 Abb., Bremen 1985.

Behrmann, Walter (1910): Zur Frage der Urstromtäler im Westen der Unterweser. - Verh. 17. dt. Geogr. Tag Lübeck 1909: 49 - 66, Berlin (Reimer) 1910.
Beijernick, W. (1931): Stuifmeelkorrels en sporen in humushoudende lagen onzer sandgronden. - Levende Nat. 35: 282 - 286, Amsterdam 1931.

- $\quad$ (1933 a): Die mikropaläontologische Untersuchung äolischer Sedimente und ihre Bedeutung für die Florengeschichte und die Quartärstratigraphie. - Proc. kon. nederl. Akad. Wetensch. 36: 107 - 115, Amsterdam 1933.

- (1933 b): De oorsprong onzer heidevelden. - Ned. Kruid. Arch. 43: 272 - 292, Amsterdam 1933.

Beug, Hans-Jürgen (1957): Untersuchungen zur spätglazialen und frühpostglazialen Floren- und Vegetationsgeschichte einiger Mittelgebirge (Fichtelgebirge, Harz und Rhön). - Flora 145: 167 - 211, 13 Abb., Jena 1957.

DECHEND, WILFRIED (1956): Der Ablauf der holozänen Nordsee-Transgression im oldenburgisch-ostfriesischen Raum, insbesondere im Gebiet von Jever i. o. - Geol. Jb. 72: 295 - 314, 4 Abb., Hannover 1956.

DüCKer, A. \& MaArleveld, G. C. (1957): Hoch- und spätglaziale äolische Sande in Nordwestdeutschland und in den Niederlanden. - Geol. Jb. 73: 215 - 234, 8 Abb., 5 Tab., Hannover 1957.

DuponT, L. M. (1986): Temperature and rainfall variation in the Holocene based on comparative palaeoecology and isotope geology of a hummock and a hollow (Bourtangerveen, the Netherlands). - Rev. Palaeobot. Palynol. 41: 241 - 271, 9 Abb., Amsterdam 1986. 
- \& Brenninkmeijer, C. A. M. (1984): Palaeobotanic and isotopic analysis of late Subboreal and early Subatlanticum peat from Engbertsdijksveen, the Netherlands. Rev. Palaeobot. Palynol. 25: 1 - 120, 10 Abb., Amsterdam 1984

DYCKERHOFF, DiRK (1927): Die deutschen Moore, ihre Nutzung und Bedeutung für die Volkswirtschaft. - Inaug. Diss. Köln, 89 S., 3 Anl., 1 Kte., Aurich (Dunkmann) 1927.

Eggelsmann, K. \& BlankenburG, J. (1990): Zur Entwicklung der Heidemoore am Nordhümmling in Niedersachsen. - Telma 20: 55 - 78, 7 Abb., 4 Tab., Hannover 1990.

ERBE, Jost (1958): Spätglaziale Ablagerungen im Emsland und seinen Nachbargebieten. - Geol. Jb. 76: 103 - 128, 13 Abb., 3 Tab., Hannover 1958.

FASTABEND, H. \& RAUPACH, F. von (1962): Ergebnisse der ${ }^{14} \mathrm{C}$ Untersuchungen an einigen Plaggenböden des Emslandes. - Geol. Jb. 79: 863 - 866, Hannover 1962.

FLORSCHÜTZ, FRANS (1941 c): Resultaten van microbotanisch onderzoek van het complex doodzand-oerzand en van daaronder en daarbroven gelegen afzettingen. - $10^{\mathrm{e}}$ wetensch. Bijeenkomst. Sect. Nederl. intern. bodenk. Ver.: 1 - 21, Groningen 1941.

Franke, Werner (1982): Das Emsland nach dem 2. Weltkrieg. - In: (Hugenberg, G., Bechtluft, H. H. \& Franke, W.): Das Emsland: 51 - 74, Leer (Rautenberg) 1982.

Freese, Johann C. (1789): Über die Vehne oder Torfgräbereien. - 174 S., 1 Abb., 1 Kt. Aurich (Borgeest) 1789 (Nachdruck Leer, Verl. Schuster, 1980).

GEeL, BAAS VAN (1978): A palaeoecological study of a Holocene peat bog section in Germany and the Netherlands. - Rev. Palaeobot. Palyn. 25: 1 - 120, 10 Abb., Amsterdam 1978.

HAvingA, A. J. (1963): A palynological investigation of soil profiles developed in cover sand. - Mededel. Landbouwhogesch. Wageningen 63, 1: 1 - 93, Wageningen 1963.

Hayen, Hajo (1979): Funde aus dem Vehne-Moor - Linie Edewecht-Bösel (Teil 1). - Archäol. Mitt. NW Deutschl. 2: 39 - 55, Oldenburg 1979.

Hugenberg, Alfred (1891): Innere Colonisation im Nordwesten Deutschlands. - Abh. staatswiss. Sem. Straßburg 8: 1 - 531, 21 Tab., 1 Kte., Straßburg 1891.

Hugenberg, Gerd (1973): Die wechselnde Bedeutung kultivierter und unkultivierter Moore in der 20jährigen Emslanderschließung. '- Telma 3: 211 - 214, Hannover 1973.

Hyszeler, G. (1947): De oudheidkundige opgravingen in Twente in de laatste jaren. - Oudheidkundig Bodenonderzoek in Nederland: 327 - 349, Amsterdam 1947.

IsENBERG, E. (1979): Pollenanalytische Untersuchungen der Vegetations- und Siedlungsgeschichte im Gebiet der Grafschaft Bentheim. - Abh. Landesmus. Naturk. Münster/Westf. 41, 2: 1 - 59, 25 Abb., Münster i. W. 1979.

JonAS, FrIEDRICH (1934): Die Entwicklung der Hochmoore am Nordhümmling. 2. Band. - FEDDE's Repert., Beih. 78, 2: 1 - 88, 24 Taf., Berlin-Dahlem 1934.

- (1935 a): Beiträge zur Emslandkunde: Klimaschwankungen des Würmglazials und Bodenbildungen des nordwestdeutschen Diluviums. - Nieders. Heimatschutz 4: 1 - 51, 15 Taf., Oldenburg i. O. 1935.

- (1938 a): Heiden, Wälder und Kulturen Nordwestdeutschlands. 1. Heft. - FEDDE's Repert. Sec. nov. Regni veg. 109, 1: 1 - 97, 48 Taf., Dahlem/Berlin 1938.

- (1941 a): Heiden Wälder und Kulturen Nordwestdeutschlands. 1. Heft. - FEDDE's Repert. Sec. nov. Regni veg. 109, 1: 1 - 97, 2: 1 - 28, 56 Taf., Berlin-Dahlem 1941.

- (1956): Das Torfprofil "Klostermoor II", Am Burlager Tief bei Papenburg. - N. Jb. Geol. Paläont., Mh. 1955: 257 - 262, Stuttgart 1956

KotTMAnN, Klaus (1963): Der Strukturwandel im Kulturlandschaftsbild des Emslandes. - Inaug. Diss., 233 S., 18 Abb., Köln 1963.

Kramm. Erich (1978): Pollenanalytische Hochmooruntersuchungen zur Floren- und Siedlungsgeschichte zwischen Ems und Hese. - Abh. Landesmus. Naturk. Münster 40, 4: 1 - 49, Münster 1978.

KuBITZKI, K. (1961): Zur Synchronisierung der nordwesteuropäischen Pollendiagramme (mit Beiträgen der Waldgeschichte Nordwestdeutschlands). - Flora 150: 43 72, 19 Abb., Kiel 1961.

LÜTTIG, GERD (1954 d): Alt- und mittelpleistozäne Eisrandlagen zwischen Harz und Weser. - Inaug., Diss., Göttingen 1952 - Geol. Jb. 70: 43 - 125, Hannover 1954.

- $\quad$ (1957 c): Geschiebezählungen als Hilfsmittel für die Erforschung des Eiszeitalters und seiner wirtschaftlich wichtigen Lagerstätten. - Umschau 57, 13: 403 - 405, Frankfurt a. M. 1. 7. 1987.

- $\quad$ (1960 a): Das Quartär im Gebiete der Struktur Bahnsen (Kr. Uelzen). - Geol. Jb. 77: 309 - 318, 2 Abb., Hannover 1960 .

- \& MaArleveld, G. C. (1962): Über altpleistozäne Kiese in der Veluwe. - Eisz. \& Gegenw. 13: 231 - 237, Öhringen/Württ. 1962

MEYER, KL.AUS-Dieter (1988): Zur geologischen Entwicklung des Emsbürener Rückens. - Hefte z. Archäol. d. Emslandes 1: 3 - 11, 4 Abb., 1 Tab., Meppen 1988.

- \& Tüxen, J. (1986): Zur geologischen Entwicklung der Papenburger Landschaft. - In. (MOHRmanN, WolfDieTER): Geschichte der Stadt Papenburg: 19 - 33, 3 Abb., Papenburg (Verl. d. Stadt) 1986.

MidDELDORP, A. (1984): Functional palaeoecology of raised bogs. - 124 p., 16 fig., Amsterdam 1984.

Müller, Helmut (1956): Ein Beitrag zur holozänen Emstalentwicklung zwischen Meppen und Dörpen auf Grund von pollenanalytischen Untersuchungen. - Geol. Jb. 71: 491 - 504, 6 Abb., 1 Tab., Hannover 1956.

Overbeck, F. \& Schmitz, H. (1931): Zur Geschichte der Moore, Marschen und Wälder Nordwestdeutschlands. I. Das Gebiet von der Niederweser bis zur unteren Ems. - Mitt. Provinzialstr. Naturdenkmalpfl. Hannover 3: 1 179, 40 Tab., Abb., Hannover 1931.

PYrITZ, EWALD (1972): Binnendünen und Flugsandebenen im Niedersächsischen Tiefland. - Gött. geogr. Abh. 61: 1 - 153, 27 Abb., 2 Ktn., Göttingen 1978.

Richter, KONRAD (1951 b): Die Entwicklungsgeschichte der Täler zwischen Lathen und Verden/Aller. - Geol. Jb. 65: 641 - 656, 5 Abb., Hannover 1951. 
Rink, Oтто (1935): Steinzeitmenschen im Tal des Langholter Tiefs. - Mein Emsland 11, 166, Papenburg 1935.

Schlicht, Elisabeth (1954): Der Hümmling in ur- und frühgeschichtlicher Zeit. I. Teil. Die Steinzeit. - Veröff. nieders. Amt Landesplang. etc. A II n. F. 24: 1 - 58, I XXIII, 5 Karten, Bremen-Horn 1954.

SCHLÜter, WolfGANG (1986): Die Vor- und Frühgeschichte im Bereich der unteren Ems. - In: (Mohrmann, WolfDieter): Geschichte der Stadt Papenburg: 35 - 89, 7 Ktn., 69 Abb., Papenburg (Stadtverl.) 1986.

SCHUCHT, Friedrich (1912): Der Lauenburger Ton als leitender Horizont für die Gliederung und Altersbestimmung des nordwestdeutschen Diltyiums. - Jb. kgl. preuß. geol. L. A. 29, 2: 130 - 150, 1 Taf., Berlin 1912.

- (1913): Zur Frage der Urstromverbindung zwischen Unterweser und Unterems. - Jb. preuß. geol. L. A. 33, 2/1: 201 - 214, Berlin 1913.
- \& Tietze, O. (1907): Das Diluvium an der Ems und in Ostfriesland. - Z. dt. geol. Ges. 59: 215 - 231, Berlin 1907.

Stefan, H. \& Dupont, L. M. (1988): Note on a programme for plotting pollen diagramms with an Atari ST microcomputer. - Pollen et Spores 30, 1: 125 - 130, Göttingen 1988.

Tietze, Oscar (1907): Über das Alter des Emstalsandes. Z. dt. geol. Ges. 59: 132 - 134, Berlin 1907.

TüXEN, Jens (1990): Grundzüge einer Geologie der Moore im niedersächsischen Flachland. - Nds. Akad. Geowiss. Veröff. 5: 5 - 21, 1 Tab., 7 Abb., Hannover 1990.

Wegener, Angela (1985): Die Besiedlung der nordwestdeutschen Hochmoore. - Telma 15: 151 - 172, 8 Abb., Hannover 1985.

Manuskript eingegangen am 15. 7. 1992 